\title{
Clinical Utility of Thrombelastography in Acute Stroke- a Current Point of View
}

\author{
Adam Wiśniewski* \\ Department of Neurology, Nicolaus Copernicus University in Toruń, Poland
}

*Corresponding author: Adam Wiśniewski, Department of Neurology, The

University Hospital No. 1, 85-094 Bydgoszcz, Skłodowskiej 9 Street, Poland.

Received Date: March 29, 2021

Published Date: April 06, 2021

\begin{abstract}
The current opinion on the clinical utility of thrombelastography in stroke was presented based on the relevant studies. The novel diagnostic tool may be be useful in prediction of early and late clinical outcome, early neurological deterioration and monitoring the safety profile among stroke subjects. Further research is needed in this field to improve the clinical utility of thrombelastography in stroke assessment.
\end{abstract}

Keywords: Thrombelastography; Stroke; Clinical utility; Clot formation; Prognosis; Safety; Efficacy

\section{Introduction}

Thrombelastography measures the dynamics of coagulation processes and clot formation in venous blood and it is estimated that this approach may be superior to conventional, static tests that assess the coagulation system [1]. Monitoring the coagulation values, in particular hypercoagulability, is widely used in many branches of medicine [2-4]. Hyperactivation of coagulation system undoubtedly constitutes a significant risk factor for ischemic stroke [5]. Therefore, the potential role of thrombelastography for diagnostic and predictive purposes is raised and increasingly attempts are made to use this innovative tool also in the field of stroke [6].

The following thrombelastography parameters are provided to evaluate the thrombosis process:

reaction time $(\mathrm{R})$ - showing the time needed to detect a clot; clots kinetics $(\mathrm{K})$ - indicating the time when a clot achieved the diameter of $20 \mathrm{~mm}$; angle (alpha) - a marker of clot growth and strengthening and maximum amplitude (MA) - reflecting the maximum firmness of a clot. The most important value related to fibrinolysis is LY30 - measuring the percentage of lysed clot after half an hour. The $\mathrm{R}$ and $\mathrm{K}$ values reflect the time of clot formation and alpha and MA are associated with its strength and enlargement. All the values have been set as a marker of hypercoagulability and their usefulness for stroke assessment and prediction were tested in several studies.

Elliot et al. [7] reported lower values of $\mathrm{R}$ and $\mathrm{K}$ parameters in stroke subjects that indicated faster clot formation compared to healthy control. Moreover, the parameters of angle and MA achieved also significantly higher values among strokes, which shows the tendency to form firm and large clots as opposed to the control. The authors emphasized the hypercoagulable status of ischemic strokes. This study was pioneering and signaled the need for further research to assess prognostic properties of thrombelastography in stroke. Yao et al. [8] measured the hypercoagulability by means of MA and revealed that severe strokes on admission (assessed in the National Institutes of Health Stroke Scale) exhibited significantly higher values of the clot maximal diameter than those observed in mild and moderate strokes. It proves an important relationship 
of stroke severity and poor early neurological condition and availability to strengthening and enlargement of thrombus. Furthermore, the authors highlighted that the maximal diameter of clot (measured in MA) was significantly elevated in stroke subjects with unfavorable functional outcome, estimated at twelve month after the onset of stroke in the modified Rankin Scale. Above finding underlines the impact of the initial firmness of clot on stroke late poor prognosis and recovery. Shi et al. [9] compared thrombelastography parameters in relation to early neurological deterioration (measured as an increase in the National Institutes of Health Stroke Scale total score over time). The authors noted that stroke subjects experienced early deterioration in clinical status achieved significantly lower values of $\mathrm{R}$, whereas the other parameters did not differ compared to stroke subjects without deterioration. It indicates that worsening in early stage of ischemic stroke might be associated with shorter time of clot detection and faster time of clot formation, whereas no relationships with parameters describing clot strength and firmness were detected.

Attempts were also made to use thrombelastography to monitor the effectiveness of reperfusion therapy [10]. However, so far no significant impact of thrombelastography values on clinical outcome of stroke subjects who underwent intravenous thrombolysis was confirmed. Nonetheless, the predictive properties were identified in estimating the risk of hemorrhagic transformation in subjects treated with alteplase. Furthermore, other authors also highlighted that thrombelastography parameters may catch the stroke subjects at increased risk of hemorrhagic complications, not related to reperfusion therapy [11]. Above findings may suggest greater use of the tool in assessing the safety compared to efficacy profile. In addition, the relationships of thrombelastography parameters with radiological findings were also raised. Kawano-Castillo et al. [12] investigated the subjects with intracranial hemorrhage and reported that longer time related to clot formation (prolonged $\mathrm{K}$ values) is associated with hematoma enlargement, which undoubtedly affect the prognosis. Shi et al. [9] analyzed relationship between thrombelastography values and new ischemic changes in diffusion-weighted imaging. The authors found that low $\mathrm{R}$ values, reflecting short time for clot formation significantly increased the new lesions visible in neuroimaging. This indirectly proves that thrombelastography may be useful in assessing the risk of progression of the neurological condition in stroke related to the extension of ischemic focus.

In my opinion the presented studies highlight the potential benefits of a comprehensive coagulation assessment measured by thrombelastography in stroke. Hypercoagulability or tendency to form solid and firm clots assessed by thrombelastography values may be useful in prediction of early and late clinical outcome, early neurological deterioration and monitoring the safety profile. Further research are needed in this field to improve the clinical utility of thrombelastography in stroke assessment.

\section{Acknowledgement}

None.

\section{Conflict of Interest}

No conflict of interest.

\section{References}

1. Mallett SV, Cox DJ (1992) Thrombelastography. Br J Anaesth 69: 307313.

2. Kang Y (1995) Thromboelastography in liver transplantation. Semin Thromb Hemost 21: 34-44.

3. McCrath D, Jerboni E, Frumento RJ, Hirsh AL, Bennett-Guerreo E (2005) Thromboelastography maximum amplitude predicts postoperative thrombotic complications including myocardial infarction. Anesth Analg 100: $1576-1583$

4. Bolliger D, Seeberger MD, Tanaka KA (2012) Principles and practice of thromboelastography in clinical coagulation management and transfusion practice. Transfus Med Rev 26: 1-13.

5. Takano K, Yamaguchi T, Uchida K (1992) Markers of a hypercoagulable state following acute ischemic stroke. Stroke 23: 194-198.

6. De Villiers S, Swanepoel A, Bester J, Pretorius E (2016) Novel Diagnostic and Monitoring Tools in Stroke: an Individualized Patient-Centered Precision Medicine Approach. J Atheroscler Tromb 23: 493-504.

7. Elliot A, Wetzel J, Roper T, Pivalizza E, McCarthy J, et al. (2015) Thromboelastography in patients with acute ischemic stroke. Int J Stroke 10: 194-201

8. Yao X, Dong Q Song Y, Wang Y, Deng Y, et al. (2014) Thrombelastrography maximal clot strength could predict one year functional outcome in patients with ischemic stroke. Cerebrovasc Dis 38: 182-190.

9. Shi Z, Zheng WC, Fu XL, Fang XW, Xia PS, et al. (2018) Hypercoagulation on Thromboelastography Predicts Early Neurological Deterioration in Patients with Acute Ischemic Stroke. Cerebrovasc Dis 46: 125-131.

10. McDonald MM, Wetzel J, Fraser S, Elliot A, Bowry R, et al. (2016) Thrombelastography does not predict clinical response to rtPA for acute ischemic stroke J Thromb Thrombolysis. 41: 505-510.

11. Yu G, Kim YJ, Jeon SB, Kim WY (2020) Thromboelastography for prediction of hemorrhagic transformation in patients with acute ischemic stroke. Am j Emer Med 38: 1772-1777.

12. Kawano-Castillo J, Ward E, Elliott A, Wetzel J, Hassler A, McDonald $\mathrm{M}$, et al. (2014) Thrombelastography detects possible coagulation disturbance in patients with intracerebral hemorrhage with hematoma enlargement. Stroke 45: 683-688. 